\title{
Sphingolipid Metabolism in Systemic Inflammation
}

\author{
H.P. Deigner, E. Gulbins, and R.A. Claus
}

\section{Introduction}

The inflammatory response - induced and regulated by a variety of mediators such as cytokines, prostaglandins, and reactive oxygen species (ROS) - is the localized host's response of the tissue to injury, irritation, or infection. In a very similar and stereotyped sequence, the mediators are thought to induce an acute phase response orchestrated by an array of substances produced locally or near the source or origin of the inflammatory response. Despite its basically protective function, the response can become inappropriate in intensity or duration damaging host tissues or interfering with normal metabolism. Thus, inflammation is the cause and/or consequence of a diversity of diseases and plays a major role in the development of remote organ failure. Better knowledge of the underlying mechanisms of these processes is, therefore, a fundamental pre-requisite fostering the molecular understanding of novel therapeutic targets or diagnostic variables.

Over the past decades, immense attempts have been made to better understand the inflammatory response at the cellular and extracellular level. In the course of these studies, a multitude of lipid mediators of inflammation, such as prostaglandins, leukotrienes, and lipoxins, has been identified and characterized. Much attention has been focused on the function of another major class of lipids, the sphingolipids, which are involved in key regulation processes such as cellular stress response and apoptosis. This chapter highlights the relevance of sphingolipids, their possible role in the regulation of the inflammatory response, and suggests key questions for further research.

\section{Sphingolipids: Structure and Function}

Sphingolipids are ubiquitous, inert membrane components of all eukaryotic cells, but are also major constituents of lipoproteins. Most of their functional properties are still being discovered, but there are at least three crucial aspects: structure, recognition, and signal transduction. Originally, sphingolipids were thought to play merely structural roles with rather inert metabolism. However, there has been a fundamental shift in the understanding of their role in modulating various cellular processes such as proliferation, differentiation, induction of apoptosis, and inflammation. The metabolism of sphingolipids comprises a set of highly regulated pathways that serve to control the levels of the individual molecule, their interconversions, and their functions. Most notable of these bioactive molecules are ceramide, ceramide-1-phosphate, sphingosine, sphingosine-1-phosphate, sphingosyl phosphorylcholine, and other derivatives (Fig. 1) [1-3]. 


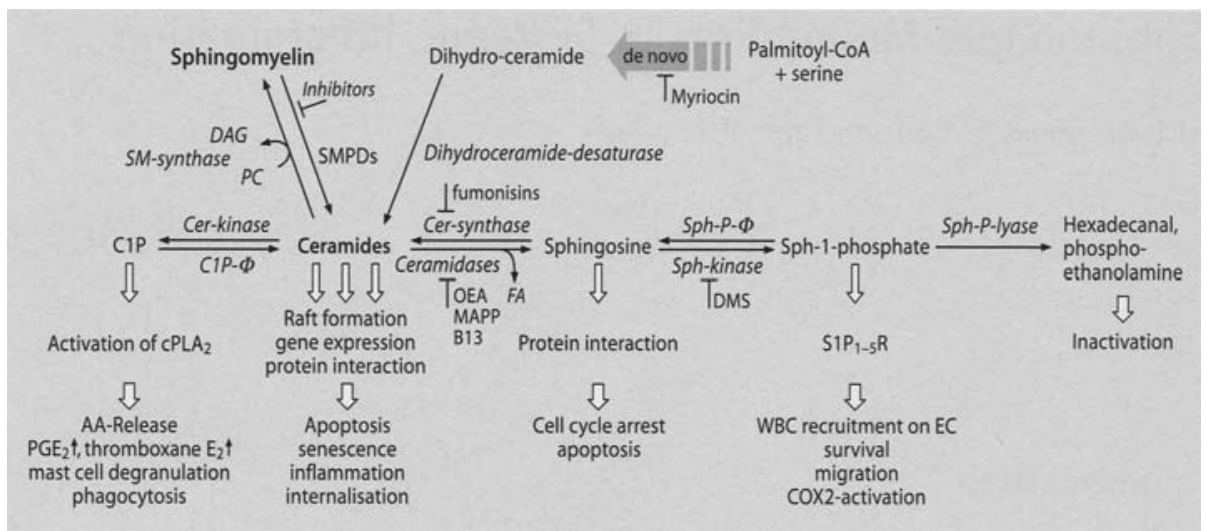

Fig. 1. Sphingolipid metabolism and interconversions. C1P: ceramide-1-phosphate; C1P- $\Phi$ : C1P-phosphatase; DAG, diacylglycerol; DMS: N,N-dimethyl-D-erythro-sphingosine; EC: endothelial cell; FA: fatty acid; MAPP: (15,2R)-D-erythro-2-(N-myristoylamino)-1-phenyl-1-propanol; PC: phosphocholine; PDMP: D-threo-1phenyl-2-decanoylamino-3-morpholino-1-propanol; $\mathrm{PGE}_{2}$ : prostaglandin $\mathrm{E}_{2}$; SMPDs: sphingomyelin phosphocholine diesterases $=$ sphingomyelinases; Sph: sphingosine; Sph-1-P: sphingosine-1-phosphate; Sph-1P- $\Phi$ : Sph-1-P-phosphatase; WBC: white blood cell.

The term 'sphingolipid' generally refers to a number of lipids consisting of a polar head group, which is attached to the primary hydroxy-moiety of ceramides. Ceramides, the central building block of all sphingolipids, consist of a sphingoid base, which is $\mathrm{N}$-acylated with fatty acids, differing in length (14-32 carbon atoms) and functionalization (degree of saturation, hydroxylation, etc.). The de novo synthesis of ceramides starts with the rate-limiting condensation of palmitoyl-CoA with serine, yielding to dihydroceramide after addition of a long chain fatty acid. The lipid mediator, resulting from desaturation, serves as a precursor for all known sphingolipids, and is further functionalized by addition of a polar head group such as carbohydrates (not referred to in this review) or phosphocholine. In eukaryotic cells, a 4,5-trans double bound is essential for bioactivity in lipids with sphingosine backbone. Sphingomyelin ( $=N$-acyl sphingosyl phosphocholin) is the other central, but inert storage pool with crucial biophysical functions, which is localized in the outer leaflet of membranes by active flip-flop mechanisms. Removal of the head group resulting in the generation of ceramide - is catalyzed by the function of several isoforms of sphingomyelinases, which are outlined in detail in Table 1. Accumulation of ceramides in cellular membranes results in the formation of lipid rafts and functional clustering of surface receptors. Another mode of action is performed by direct interaction with proteins in the activation of kinases (e.g., protein kinase $C_{\text {, iso- }}$ form $\zeta$ ) coupling the action of ceramide to activation of transcription factor, nuclear factor-kappa B (NF-kB) and stress activated protein kinases. In addition, the activation of phosphatases or cathepsin D result in signaling, which enables stimulation of adequate and fine tuned cellular responses. For ceramide clearance, ceramidases hydrolyze the $\mathrm{N}$-acyl fatty acid, resulting in the generation of sphingosine, which can be phosphorylated at the primary hydroxy-moiety by isoforms of sphingosinekinase (SphK) forming sphingosine-1-phosphate (S1P). S1P is cleaved by S1P lyase in an irreversible manner, whereas ceramides or sphingosine can be reversible synthesized by the action of S1P-phosphatase or ceramide synthase, respectively. A 
Table 1. Sphingomyelin hydrolyzing enzymes. In human tissues, five RefSeq validated proteins have so far been described, differing in pH-optima, cellular localization and functions. SMPD1 (sphingomyelin phosphodiesterase 1) codes for the acidic sphingomyelinase, which is either localized in lysosomes by mannose-6phosphate-receptor/sortilin-dependent trafficking or plasma secreted due to pro-inflammatory stimulation. SMPD2 codes for a neutral sphingomyelinase-1 (NSM1); however SMPD3 is the bona fide gene for NSM2 [83]. A variety of isoform specific inhibitors is described in the right column.

\begin{tabular}{|c|c|c|c|c|c|}
\hline $\begin{array}{l}\text { HUGO name } \\
\text { (Genbank ID) }\end{array}$ & $\mathrm{pH}$ & cofactor (s) & Localization & Function & Inhibitor(s) \\
\hline $\begin{array}{l}\text { SMPD1 } \\
\text { (NM_000534) }\end{array}$ & $\sim 5$ & none & $\begin{array}{l}\text { lysosomes, } \\
\text { caveolae, } \\
\text { platelets }\end{array}$ & $\begin{array}{l}\text { Metabolic degradation of } \\
\text { sphingomyelin; induction of } \\
\text { apoptosis, differentiation, } \\
\text { inflammation. } \\
\text { Congenital deficiency: Nieman- } \\
\text { Pick Disease }\end{array}$ & $\begin{array}{l}\text { desipramine, } \\
\text { SR33557, } \\
\text { NB6, DTT }\end{array}$ \\
\hline $\begin{array}{l}\text { SMPD1 } \\
\text { (NM_000534) }\end{array}$ & $5-7$ & $\mathrm{Zn}^{++}$ & $\begin{array}{l}\text { plasma } \\
\text { secreted } \\
\text { enzyme }\end{array}$ & $\begin{array}{l}\text { Atherogenesis, hemophagocytic } \\
\text { lymphohistiocytosis, diabetes, } \\
\text { ARDS, sepsis }\end{array}$ & \\
\hline $\begin{array}{l}\text { SMPD2 } \\
\text { (NSMI) } \\
\text { (NP_003071) }\end{array}$ & $6.5-7.5$ & $\mathrm{Mg}^{2+}$ & $\begin{array}{l}\text { membrane } \\
\text { (multi-pass } \\
\text { membrane } \\
\text { protein) }\end{array}$ & $\begin{array}{l}\text { Differentiation, permeability } \\
\text { barrier function }\end{array}$ & \\
\hline N.N. & $\sim 7$ & $\begin{array}{l}\text { Triton X-100, } \\
\text { deoxycholat, } \\
\text { phosphati- } \\
\text { dylserine, } \\
\text { (in-vitro) }\end{array}$ & cytoplasma & $\begin{array}{l}\text { Differentiation, } 1,25-\text { Dihydroxy- } \\
\text { vitamin } D_{3} \text {, IFN } \gamma \text { and TNF- } \alpha \\
\text { induced signal transduction in } \\
H L-60 \text { cells (no RefSeq validati- } \\
\text { on) }\end{array}$ & $\begin{array}{l}\mathrm{Cu}^{2+}, \mathrm{Fe}^{3+}, \\
\mathrm{Zn}^{2+}\end{array}$ \\
\hline $\begin{array}{l}\text { SMPD3 } \\
\text { (NSMII) } \\
\text { (NP_061137) }\end{array}$ & $\sim 7$ & $\begin{array}{l}\mathrm{Mg}^{2+}, \mathrm{Mn}^{2+} \\
\text { (millimolar); } \\
\text { phosphatidyl- } \\
\text { serine, } \\
\text { arachidonate }\end{array}$ & membrane & $\begin{array}{l}\text { Hydrolysis of membrane bound } \\
\text { SM; differentiation, prolifera- } \\
\text { tion, inflammation, apoptosis, } \\
\text { lung injury; } \\
\text { bona fide gene }\end{array}$ & $\begin{array}{l}\text { scyphostatin, } \\
\text { glutathione, } \\
\text { manumycin, } \\
\text { ubichinol, } \\
\text { chloro-genti- } \\
\text { sylchinone, } \\
\text { GW4869, } \\
\text { C11AG }\end{array}$ \\
\hline $\begin{array}{l}\text { ENPP7 } \\
\text { (NP_848638) }\end{array}$ & $8.0-9.5$ & bile salts & $\begin{array}{l}\text { intestinal } \\
\text { lumen; bile, } \\
\text { mucosal } \\
\text { membrane; }\end{array}$ & $\begin{array}{l}\text { degradation of dietary sphino- } \\
\text { gomyelin; intestinal PAF-degra- } \\
\text { dation, role in intestinal inflam- } \\
\text { mation or inhibition of colonic } \\
\text { tumorigenesis? }\end{array}$ & \\
\hline
\end{tabular}

ARDS: acute respiratory distress syndrome; DTT: dithiothreitol; HUGO: Human Genome Organization; IFN $\gamma$ : interferon gamma; PAF: platelet activating factor; TNF- $\alpha$ : tumor necrosis factor alpha.

major metabolite of ceramide is ceramide-1-phosphate (C1P), which is formed by direct phosphorylation of ceramide by the action of a specific kinase. C1P plays a critical role regulation proliferation, differentiation, apoptosis and generation of eicosanoids via direct binding to target proteins, e.g. phospholipase $A_{2}$. There is also increasing evidence of a key role in phagocytosis [4], and most importantly, inhibition of SMPD1 by physical interaction has been observed [5]. 
A major principle in understanding ceramide metabolism (which applies to all other bioactive lipids) is the distinct subcellular localization and topology, which is outlined, e.g., in a review by van Meer and Lisman [6]. In recent years, considerable attention has been given to other types of sphingosine base derivatives, namely 'lyso'-types or $N$-methyl derivatives, which are highly bioactive, however little is known about their origins or function(s).

Beyond the stimulus-dependent activation of sphingolipid metabolism, considerable evidence has been gathered regarding the role of subcellular compartmentalization of the generation and accumulation of individual metabolites. Numerous studies point to a tightly controlled localization of enzyme and lipid pools within the cell, e.g., suggesting a specific, apoptogenic role for endogenously generated ceramide from mitochondrial membranes [7]. Within the different compartments, distinct isoforms of sphingomyelinases perform specific functions via generated ceramide, such as inhibition of protein kinase $C$ translocation, inhibition of NF- $\kappa B$, and aggregation of the Fas receptor $[6,8]$. Another mode of action is the endosomal generation of ceramide by acid sphingomyelinase as a direct activator of cathepsin D [9]. Considering that de novo synthesis of ceramide is mainly restricted to the endoplasmatic reticulum as well as to membranes associated with mitochondria and the nucleus, the localization and the effects of synthesis inhibitors, such as fumonisins, suggest a pivotal role for ceramide subfractions in mediating apoptosis. Furthermore, recent evidence has indicated the contribution of the mitochondria and the nucleus as major sites in the initiation of apoptosis by ceramide [10].

Due to the dynamic equilibrium of lipid mediators, cells may respond to an extracellular stimulus with perturbation of the balanced 'rheostat' as a consequence of activation of sphingomyelinases followed by accumulation of ceramide and related metabolites. For a better understanding of the sphingolipid flux during the cellular stress response as well as the effects of pharmacological or molecular manipulations of critical enzymes (see below), it is imperative to use reliable measuring systems. Recent molecular advances in the identification of enzymes involved in sphingolipid metabolism and improvements in the analytical equipment, e.g., mass spectroscopy, have facilitated a better understanding of the role and function of sphingolipids [11].

\section{Step by Step: The Hydrolysis of Sphingomyelin}

Sphingolipid metabolism is a constitutive process beginning with removal of the head group, phosphocholine, yielding the intermediate product and lipid mediator, ceramide, a reaction catalyzed by a family of enzymes termed sphingomyelinases (or systematically sphingomyelin phosphocholine diesterase, SMPD). The isoforms are distinguished by different $\mathrm{pH}$-optima, localization and cation dependence (Table 1) [12]. Of the five human sphingomyelinases identified so far, the neutral $\mathrm{Mg}^{2+}$-dependent and the lysosomal acid isoform are the most thoroughly studied and, therefore, seem to appear most relevant for generation of ceramide during the stress response $[12,13]$.

The activity of a neutral $\mathrm{pH}$-optimum, $\mathrm{Mg}^{2+}$-dependent sphingomyelin hydrolyzing enzyme was initially described four decades ago [14]. The maintenance of this isoform's specific activity in both the SMPD1 ${ }^{-1-}$ model as well as in cells obtained from patients affected with Niemann-Pick disease Type A, verified that these isoforms are distinct products of different genes [15]. The purified enzyme, termed 
neutral sphingomyelinase 1 (NSMI), exhibits an optimal $\mathrm{pH}$ at 7.4 , an estimated molecular mass around $60 \mathrm{kDa}$, and a specific activity for hydrolysis of sphingomyelin, but not phosphatidylcholine. The presence of divalent cations such as magnesium or manganese as well as a proper lipid composition containing anionic lipids and unsaturated fatty acids, especially phosphatidylserine and arachidonic acid, are essential for its activity. Recently, it was reported that in caveolae-enriched membrane fractions derived from bovine lung microvascular endothelial cells, a caveolar isoform of neutral sphingomyelinase cross-reacts with specified antibodies against an isoform with a higher molecular weight purified from brain [16].

Two years after the cloning of NSMI, the sequencing and characterization of another mammalian, brain-specific $\mathrm{Mg}^{2+}$-dependent sphingomyelinase, NSMII, was reported [17]. NSMII is also activated by phosphatidyl-serine and other anionic phospholipids, suggesting an enrichment of the protein in the inner leaflet of the plasma membrane, at the mitochondria as well as the endoplasmatic reticulum. Using deletion mutants of the p55 tumor necrosis factor (TNF) receptor, a neutral sphingomyelinase activation domain (NSD) was identified, which is juxtaposed to the death domain of the TNF receptor. A corresponding protein, termed 'factor associated with neutral sphingomyelinase activation' (FAN), was described which binds to NSD, resulting in a functional coupling of the TNF receptor with neutral sphingomyelinase activation [18]. Results demonstrating an interaction of neutral sphingomyelinase with activated C-kinase 1 as well as caveolin-1 further suggest that the formation of multiprotein complexes are involved in the signal transduction from TNF- $\alpha$ to neutral sphingomyelinase, thus regulating its activity [19]. Most recently, it was demonstrated that hydrogen peroxide $\left(\mathrm{H}_{2} \mathrm{O}_{2}\right)$-induced apoptosis of endothelial cells was completely blocked in NSMII-loss of function models [20], highlighting the essential role of NSMII in the induction of apoptosis.

Recent studies have shown that SMPD1 has characteristics of a lysosomal and secretory sphingomyelinase, which both derive from the same gene (smpdl) exhibiting differences in the oligosaccharide structure and $N$-terminal proteolytic processing with subsequent differential protein trafficking $[21,22]$. As a result the proteins are present in both blood plasma and intracellular lysosomes [12]. Previous studies suggested that the lysosomal mannose-6-phosphate receptor is implicated in SMPD1 trafficking [23]. However, the type I transmembrane glycoprotein, sortilin, is also involved in targeting of the protein: Truncated sortilin partially inhibits lysosomal trafficking and enhances the secretion of SMPD1 [24]. Among the known types of eukaryotic sphingomyelinases, only the secretory variant, SMPD1, has so far been shown to be responsible for extracellular hydrolysis of membrane and lipoprotein bound sphingomyelin [22]. SMPD1 is secreted by macrophages, human skin fibroblasts, and human vascular endothelial cells; the latter are assumed to be the chief source of the enzyme [12,25]. In endothelial cells, apical as well as basolateral secretion of SMPD1 is stimulated by a variety of pro-inflammatory mediators, including interleukin (IL)-1 $\beta$, interferon (IFN) $\gamma$, IFN $\beta$, TNF- $\alpha$, platelet activating factor (PAF), and ROS as endogenous mediators, as well as endotoxin as an exogenous mediator [25]. The secreted form of SMPD1 is stimulated by zinc-ions, whereas the lysosomal form is already tightly bound to the cation. Thus, the source of the activity can be distinguished as originating, e.g., from damaged and disintegrating endothelium or from activated cells driven by a pro-inflammatory impetus. An increase to $\mathrm{pH} 7.4$ which is far beyond the optimum for the lysosomal protein as found in plasma, appears to affect only the substrate affinity (i.e., the $K_{m}$ ), but not the activity $\left(V_{\max }\right)$ of SMPD1 [24], a fact important in estimating the extralysosomal activity of the enzyme [21,27]. 
In the following section, cellular mechanisms of the action of sphingolipid mediators are briefly discussed.

\section{Receptor-mediated Effects}

The lysosphingolipid, S1P, functions as a ligand for at least five G-protein species coupled to cell surface receptors termed $S 1 P_{1-5} R$ regulating cell proliferation, apoptosis and motility. Activation by S1P binding results in sequestering of lymphocytes from the circulation to lymph nodes and Peyer's patches. This redistribution effectively reduces $T$ cell numbers at the sites of inflamed tissue or graft sites. TNF- $\alpha$ induced sphingosine kinase 1 (SphK1) activity is followed by increased S1P levels and subsequent cyclooxygenase (COX)-2 activation. The inhibition of S1P clearance, e.g. by targeting S1P lyase or S1P phosphatase, augments COX-2 activation and consequently prostaglandin (PG)E2 generation. Exogenous S1P addition dose-dependently reproduced COX-2 induction observed subsequent to TNF- $\alpha$ addition. In neutrophils, the hydrolysis of sphingomyelin increased SphK activity, and S1P generation appears to be a key event in neutrophilic priming by TNF- $\alpha$ and other stimuli $[28,29]$. During activation of mast cells, S1P levels increase resulting in the release of inflammatory mediators such as leukotrienes and cytokines. In contrast, sphingosine has an opposing effect on mast cell activation [30].

Cell migration is crucial to the proper functioning of the cells involved in the course of inflammatory processes. The SphK/S1P pathway plays an important role in chemoattractant signaling in myeloid differentiated HL-60 cells [31]. In addition, S1P was found to act as a specific and effective regulator of migration of freshly isolated human neutrophils across endothelial cells [29]. This transmigration, which is essential for the recruitment of white blood cells to the site of inflammation, is enabled by the expression of adhesion molecules, such as intracellular adhesion molecule (ICAM)-1, on endothelial cells.

Interest in sphingolipids as signaling molecules in immune cells increased as it became evident that sphingosine, as well as ceramide, induces apoptosis in $\mathrm{T}$ cells, whereas S1P emerged as a counterregulatory principle; in Th2 cells sphingosine (but not ceramide) exerts inhibiting effects on proliferation, implying that individual immunological responses depend on the dynamic balance of sphingolipids [2].

\section{Raft Formation: Ceramide-induced Reorganization of Membrane Receptors}

There is currently no defined receptor for ceramide; however, the lipid has been shown to be involved in signal transduction by altering membrane organization and fluidity. Ceramide has the tendency to self-associate and to form ceramide-enriched microdomains that spontaneously fuse to large ceramide-enriched macrodomains, also termed ceramide-enriched platforms. These biophysical properties act to reorganize very small distinct domains in the cell membrane, termed rafts, which serve in the spatial organization of signaling molecules. Thus, ceramide enriched membrane platforms have been shown to mediate clustering (i.e., tighter packing) and recruitment of signaling molecules, while excluding others, and to reorganize the topology of proteins and aggregation of receptors inducing the transduction of signals. 
These specialized domains of the cell membrane are central for the spatial organization of receptors and signaling molecules. Upon stimulation, acid sphingomyelinase is translocated to the outer leaflet of the cell membrane, apparently mediated by a fusion of sphingomyelinase containing vesicles with the cell membrane resulting in the cell surface exposure of the enzyme. Generated ceramide then contributes to the formation of both small and large rafts, ceramide-enriched platforms, which in turn may amplify signaling in response to stress, irradiation, ultraviolet light, gamma irradiation, doxorubicin, cisplatin, disruption of integrin-signaling, TNF receptor, CD40, LFA-1, DR5/TRAIL, CD20, Fc $\gamma$ RII, CD5, LFA-1, CD28, TNF, IL-1 receptor, PAF-receptor, and CD14 [32].

CD95-dependent apoptosis requires a pre-association of CD95, the formation of the death-inducing signaling complex (DISC), and clustering of CD95 in specific membrane domains. In this context, the acid isoform, activated upon CD95 ligation and initial caspase 8 activation, is translocated and functions upstream of the DISC to mediate CD95 clustering in ceramide-enriched membrane platforms, an event required for DISC formation, yielding full caspase 8 activity and apoptosis [33].

Identical mechanisms seem to be operative in the signaling of apoptosis by other death receptors or stress suggesting a general role of ceramide-enriched platforms in apoptosis and explaining the function of SMPD1 and ceramide in multiple signaling pathways $[34,35]$.

The metabolic precursor, sphingomyelin, is important for Fas receptor clustering through aggregation of lipid rafts, leading to Fas-mediated apoptosis. Experiments with sphingomyelin synthase (SMS)-defective WR19L cells transfected with the human Fas gene (WR/Fas-SMS ${ }^{-i}$ ), and cells that have been functionally restored by transfection with SMS1 (WR/Fas-SMS1), show that expression of membrane sphingomyelin enhances Fas-mediated apoptosis through increasing efficient translocation of Fas into lipid rafts, Fas clustering, DISC formation, and subsequent activation of caspases [34].

\section{Role of Sphingolipids in Regulation of the Immune Response, Susceptibility to Infection, and Triggering of Pathogen-associated Apoptosis}

Some pathogens activate SMPD1, which releases ceramide in membrane rafts, structures which enable a host/pathogen interaction by formation of negative membrane curvatures. An abundance of evidence indicates that the formation of ceramideenriched membrane raft structures facilitates the invasion of various pathogens [37-39]. Often, the final result of ceramide-mediated cellular entry is containment and/or inactivation of the pathogen. The importance of ceramide in pathogen invasion is underscored by studies investigating Neisseria gonorrhoeae, Pseudomonas aeruginosa, Staphylococcus aureus and Sindbis virus, which have been shown to activate SMPD1 resulting in rapid ceramide formation. The strength of these studies was the demonstration that inactivation of SMPD1 nearly completely hindered pathogen internalization. As a result, all pathogen-associated alterations of membrane scaffold have been shown to mediate internalization of bacteria, viruses, and parasites into the host cell, to initiate apoptosis of the host cell upon infection, and to regulate the release of cytokines from infected mammalian cells [37]. On the other hand, ceramide-enriched membrane platforms are also central to the host defense against potentially lethal pathogens like $P$. aeruginosa which, upon infection, trig- 
gers activation of SMPD1 and the release of ceramide in sphingolipid-rich rafts within minutes [39]. Failure to generate ceramide-enriched membrane platforms in infected cells results in an unabated inflammatory response, such as massive release of IL-1 $\beta$ and septic death in mice.

In addition, the protozoan, Leishmania donovani, was shown to induce ceramide formation by both de novo synthesis and SMPD1 activation, resulting in elevated ceramide levels which facilitate the survival of the parasite in the intramacrophageal milieu [41]. Rafts, in addition to playing a crucial role in pathogen entry, have also been shown to serve as platforms for viral assembly or budding and in the intracellular trafficking of phagosomes [42]. It has also been shown that ceramide-enriched membrane platforms are involved in the infection of human cells with pathogenic rhinoviruses [42]. The infection of human epithelial cells with rhinovirus strains triggers a rapid activation of SMPD1, the formation of ceramide in the cell membrane and, finally, the formation of large ceramide-enriched membrane platforms. These events correlate with microtubule- and microfilament-mediated translocation of the enzyme from an intracellular compartment onto the extracellular leaflet of the cell membrane. In agreement with a key role of SMPD1 and ceramide in the infection of human cells with rhinoviruses, genetic deficiency or pharmacological inhibition of the SMPD1 prevented infection of human epithelial cells by rhinoviruses.

The susceptibility to infections of individuals with these diseases, and with elevated plasma levels of SMPD1, which has been observed during the course of sepsis and systemic inflammation (see below), might contribute to an altered immune response rendering individuals susceptible to other, secondary infections, e.g. from colonizing bacteria. All these studies support the notion that rafts and ceramideenriched membrane platforms function as central structures involved in the infection of mammalian cells by pathogens and as targets for the development of antiinfective drugs.

\section{Role of the Oxidative Balance}

The tripeptide, glutathione (GSH), plays a major role in cellular redox homeostasis. Several lines of evidence suggest that ROS, such as superoxide radical $\left(\mathrm{O}_{2} \cdots\right), \mathrm{H}_{2} \mathrm{O}_{2}$, and the hydroxyl radical $(\cdot \mathrm{OH})$, as well as reactive nitrogen species, such as the peroxide radical $\left(\cdot \mathrm{ONOO}^{-}\right)$and the cellular redox potential, which is mainly regulated by GSH concentration, are tightly linked to the regulation of sphingolipid hydrolysis.

NSMI activity requires the presence of reducing agents, and recent studies have shown that it is reversibly inhibited by ROS and oxidized glutathione, whereas it is irreversibly inhibited by peroxynitrite [43]. As described above, NSMII activity and trafficking are tightly regulated by the oxidative intracellular status in a complex dynamic process [20]. On the other hand, sphingolipids are also known to play an important role in maintaining cellular redox homeostasis through regulation of plasma membrane oxidants, such as NADPH oxidase, with subsequent disturbance of mitochondrial integrity and induction of apoptosis [44]. Post-translationally, both recombinant and plasma borne SMPD1 are also directly activated in the presence of oxidizing agents by modification either of a 'cysteine switch' or by a copper induced dimerization via disulfide bond formation responsible for an increase in enzymatic hydrolysis $[45,46]$. It is, therefore, tempting to speculate that at least some of the above-named stress stimuli stimulate SMPD1 via redox processes. Ceramide itself is 
known to trigger the release of ROS, from, for example, endothelial cells [47]. Thus, under oxidative conditions, it might be part of a self-perpetuating and positive feedback mechanism for the post-translational activation of SMPD1. Interestingly, in neutrophils, ceramide generation, CD95 clustering, and apoptosis were dependent on ROS suggesting that an altered redox status initiates ligand-independent death receptor signaling via activation of SMPD1 and clustering of preformed DISC components in lipid rafts [48].

\section{Increased SMPD1 Activity: Cause or Consequence in Organ Failure?}

A 2-3-fold increase in plasma sphingolytic activity has been observed in animal models after application of endotoxin or pro-inflammatory cytokines (TNF- $\alpha$, IL$1 \beta)$ [49-51]. In a human setting, there is indirect evidence for altered SMPD1 activity during systemic disease. An increased ceramide/sphingomyelin ratio has been reported in septic patients as well an association with a poor clinical outcome [52]. At a cellular level, increased ceramide concentrations have been reported in circulating mononuclear cells of septic patients that were positively correlated with plasma TNF- $\alpha$ levels [53]. In this study, patients with multiple organ failure (MOF) exhibited a more pronounced ceramide accumulation. Furthermore, raised SMPD1 levels have also been reported in children with hypercytokinemia due to hemophagocytic lymphohistiocytosis [54]. In order to achieve a deeper insight into the functional role of SMPD1 during sepsis, we analyzed its presence and activity in patients with various degrees of different concomitant diseases, variable sources of infection, and a broad range of disease severity as reflected by differing levels of inflammatory markers. Circulating SMPD1 was found to be markedly elevated on the first day of sepsis. During the course of the disease, an inverse trend between survivors (decrease) and non-survivors (further increase) as well as a positive association with organ failure scores was observed [45].

The first clinical implications for inhibition of sphingolipid hydrolysis came from studies dealing with the lysosomal activity of SMPDI in leukocytes obtained from patients with major depression [55]. In addition to an increase in the constitutive activity in peripheral blood mononuclear cells dependent on illness severity, ex vivo treatment of the cells with tricyclic antidepressive drugs, such as amitryptiline or imipramine, resulted in a rapid reduction in SMPD1 activity. As reported above, ceramide generation changes the composition of membrane structures thus mediating the formation of platforms, which facilitate receptor clustering and signaling. Thus, alterations in SMPD1 activity may have clinical implications for the regulation of serotonin and dopamine reuptake transporter activity. The pathophysiological significance of altered SMPD1 activity in major depression remains to be further elucidated. The observed increase, however, supports the concept that SMPD1 activity and ceramide generation may function in these molecular phenomena, may contribute to subsequent interference with other enzymes such as phospholipase $A_{2}$ and isoforms of protein kinase $\mathrm{C}$, as well as synaptic transmission. It is, therefore, tempting to speculate that SMPD1 activity may be a molecular target for antidepressant drug therapy.

There is also an increasing body of evidence that extracellular hydrolysis of sphingomyelin due to secreted SMPD1 activity may be involved in mediating systemic effects. Induced by the massive release of cytokines and other factors into the circulation, this phenomenon, termed generalization, leads to functional effects 
occurring outside the genuine locus of actual infection or treatment, which is often observed in the pathogenesis of MOF or subsequent to radiotherapy for various malignancies. Sathishkumar et al. demonstrated, in patients who underwent high dose spatially fractionation radiation, an elevation of both the level of plasma secreted SMPD1 activity and the concentration of lipoprotein bound ceramide, with a correlation of SMPD1 activity and clinical outcome [56]. Most importantly, a role of SMPD1 and ceramide in the generation of PAF-mediated pulmonary edema was shown by Goggel and colleagues [57]. In an animal study, it was clearly demonstrated that SMPD1 plays a critical role both locally in ceramide generation in the stimulated tissue as well as in the increase of vascular permeability resulting in edema formation. Accumulation of ceramide was paralleled by synthesis and release of aspirin-inhibitable prostaglandin production. SMPD1 deficient mice exhibited $50 \%$ less pulmonary edema than wild type animals. Consistent with these findings, agents interfering with ceramide generation as well as anti-ceramide antisera reduced edema formation initially triggered by exogenously administered PAF, bacterial endotoxin, or intratracheal instillation of acid. The latter two models are relevant to the increased permeability during lung edema observed in the clinical condition of sepsis or aspiration-induced pneumonia, which are common and often lethal precipitators of acute lung injury (ALI) in humans [58]. On the other hand, systemic effects were proven by the release of the enzyme into circulation via venous efflux enabling extra-pulmonary ceramide generation at the outer leaflet of remote endothelial membranes [57]. In this context, it is noteworthy that until now plasma secreted SMPD1 is the only enzyme shown to be responsible for extracellular sphingomyelin hydrolysis; activity of NSMII or other sphingomyelinase isoforms has not been observed in plasma $[49,57]$. Additionally, SMPD1 translocates to cell surfaces and has activity in the outer leaflet of the cellular membrane; the membrane-associated enzyme may behave differently to recombinant protein administered in solution [34]. However, it is not yet clear whether the enzyme translocated onto the plasma membrane upon CD95 stimulation [33] is identical to the form described by Tabas as the plasma secreted isotype [21].

Signaling by ceramide is also critically involved in molecular mechanisms triggering ischemia/reperfusion injury as well as TNF- $\alpha$ induced organ damage. In the liver, ceramide levels transiently increased in galactosamine/TNF- $\alpha$-induced liver damage or after the reperfusion phase of ischemia due to early activation of hepatic SMPD1. Inhibition of sphingolytic activity decreased ceramide generation and subsequent increase in surrogates for tissue damage, hepatocellular apoptosis, and mitochondrial targeting of apoptosis-triggering gangliosides, resulting in cytochrome $c$ release $[59,60]$. Thus, modulation of sphingolipid signaling may be of therapeutic relevance, e.g., in hepatic tissue injury.

In addition, thrombin activated thrombocytes release SMPD1 [61] and these cells may be a candidate source for SMPD1 because they are critically involved in triggering thrombotic and inflammatory events, e.g., resulting in lung edema [58]. By triggering Weibel-Palade body exocytosis, ceramide activated endothelial cells release von Willebrand factor and P-selectin, which induce leukocyte rolling as well as platelet adhesion and aggregation [62]. This phenomenon, which was also observed after addition of sphingomyelinase, suggests a novel and intriguing mechanism by which ceramide may contribute as an intermediary to vascular inflammation raising a thrombophilic state. A hypothetical schema of the functions of plasma secreted SMPD1 and subsequent ceramide generation during systemic inflammation and infection is outlined in Fig. 2. 


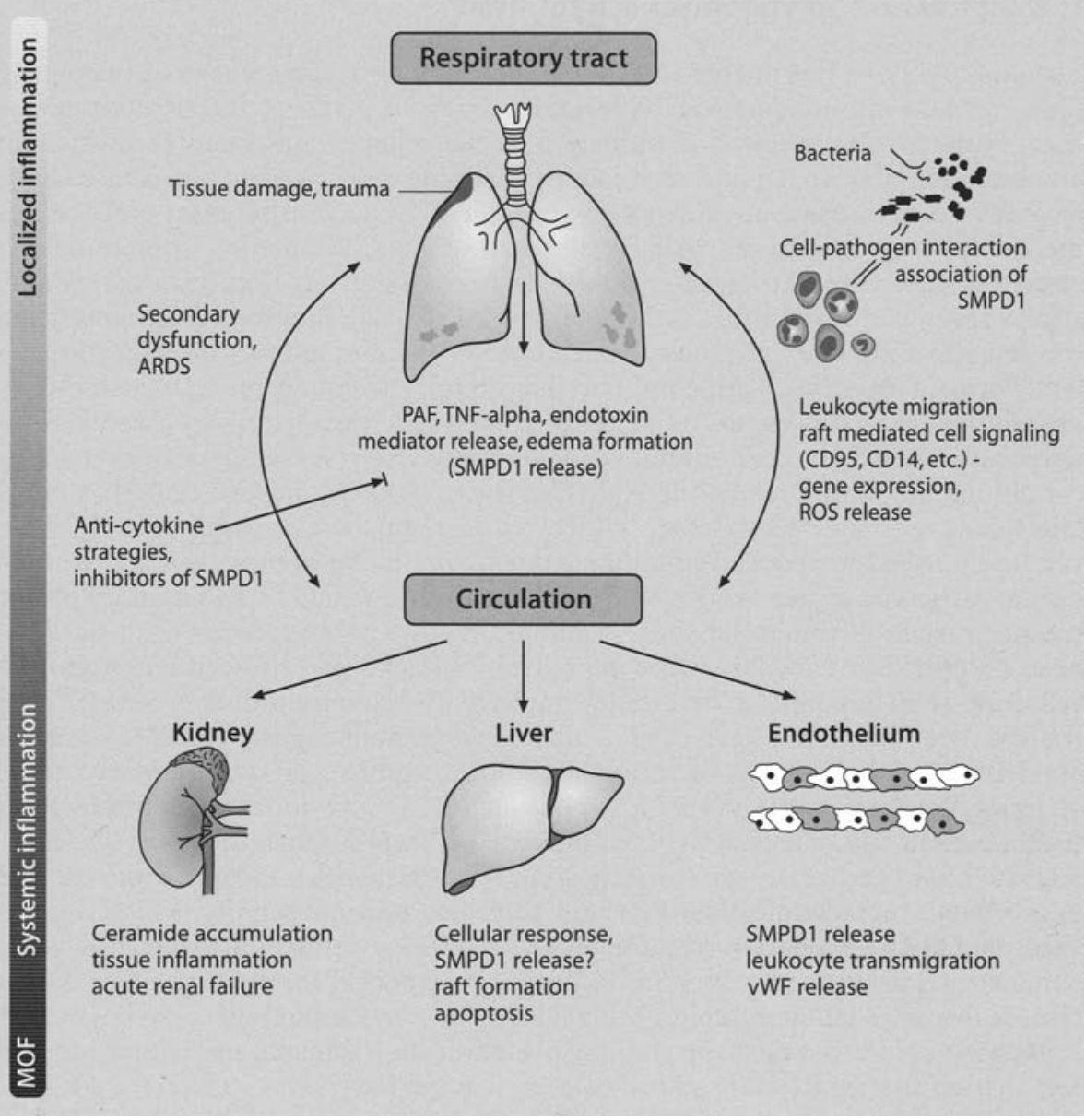

Fig 2. Plasma secreted sphingomyelinase in inflammation. Hypothesized impact of sphingomyelin phosphocholine diesterase (SMPD)1 in the development and exacerbation of systemic inflammation. ARDS: acute respiratory distress syndrome; WWF: von willebrand factor; MOF: multiple organ failure.

It is noteworthy that the observed plasma activities in patients with inflammation of different origins clearly exceed the enzymatic activity reported to induce biological effects in cell culture systems [63, 64]. SMPD1 has been shown to hydrolyze sphingomyelin in low density lipoprotein (LDL) particles [27], which is important considering that most of serum sphingomyelin and nearly half of the ceramides are known to be localized in circulating LDL [49]. Therefore, the accumulation of ceramide in both circulating cells and in lipoproteins may serve as a persistent reference pool reflecting elevated plasma secreted SMPD1 activity. Though not proven, it might, therefore, be possible to discriminate between transient/short-term effects and long lasting/even mild variations by determination of enzyme activity levels in a functional assay or resulting ceramide accumulation. According to our own observations and data from other groups, there is only marginal degradation of plasma ceramide, resulting in the mentioned accumulation of the mediator in lipophilic compartments, such as plasma lipoproteins. 


\section{Inhibitors of Sphingomyelin Hydrolysis}

The availability of specific pharmacological inhibitors of some enzymes of sphingolipid metabolism and ongoing molecular cloning of some of the key enzymes of these pathways, has allowed examination of the cellular consequences of inducing accumulation of endogenous ceramides or ceramide clearing enzymes. These studies have provided substantial evidence for the role of sphingomyelinases and the lipid mediator, ceramide, in initiating cellular responses. Numerous compounds are known to inhibit NSMI. Structural analogs of the naturally occurring compounds, scyphostatin and manumycin A, have been used [65, 66]; however, these compounds contain a reactive epoxide moiety, which enables them to interact directly and covalently with a variety of proteins, thus hampering the interpretation of biological experiments. For this reason, a panel of compounds was synthesized containing a polyunsaturated fatty acid bound to a chemically less reactive, under physiological conditions, 1,2-amino alcohol derivative with a cyclohexenone moiety [65]. A redoxdependent reversible mechanism is involved in regulation of NSMI activity, which can be abolished by reduced glutathione during induction of programmed cell death due to ischemia in neuronal cells [13]. Structural analogs of sphingomyelin with reactive structural moieties, such as difluoromethylenephosphonic acid, have also been described as effective, non-competitive inhibitors of TNF- $\alpha$-induced neuronal cell death [67]. In addition, various low molecular weight inhibitors of SMPD1 activity have been identified, including 5'-adenosine monophosphate (5'-AMP); tricyclic antidepressive drugs, such as imipramine, amitryptiline, or desipramine; cationic amphiphilic drugs, such as NB6, L- $\alpha$-phosphatidyl-D-myo-inositol-3,5-bisphosphate (PtdIns3,5P2), and phosphatidyl-myo-inositol 3,4,5-triphosphate [PtdIns $(3,4,5) \mathrm{P}(3)]$; SR33557; and derivatives of $\alpha$-mangostin. Tricyclic antidepressants induced rapid intracellular degradation of SMPD1 and abolished enzyme activity, which could be abrogated by preincubation with the protease inhibitor, leupeptin. Interestingly, data obtained using plasmon resonance technology supported the concept of an interaction of the amphiphilic inhibitor with the enzyme and immobilized sphingomyelin containing lipid bilayers, displacing the protein from its membrane bound substrate and rendering it susceptible to proteolytic cleavage [68].

In an experimental model of endotoxic shock, specific inhibition of SMPD1 by the carbazol derivative, NB6, resulted in decreased hepatocellular apoptosis and improved survival rate, providing further evidence for a crucial role of SMPD1 in the pathogenesis of systemic inflammation and subsequent organ failure [45]. Remembering that SMPD1 fulfils crucial functions in host defense, a non-beneficial effect of SMPD1 inhibition during infection of mice with living bacteria in a model of polymicrobial peritoneal contamination and infection with overwhelming mortality after instillation of human feces is feasible (Bunck et al., unpublished data).

FTY720, a substrate for SphK has shown tremendous promise as a regulator of multiple levels of inflammation. FTY720 is chemically derived from myriocin, an ascomycete metabolite, and is metabolized to the phosphor-derivative, FTY-P, by sphingosine kinases to become active as an S1P agonist at four of the five known S1P receptors [69]. FTY720 has been tested as an immunomodulator in renal transplant patients and patients with emphysema. The Sph1P mimetic produces lymphopenia by reducing recirculation of lymphocytes and sequestering them into lymph nodes. FTY-P induces $S 1 P_{1} R$ internalization of lymphocytes, which abrogates the interaction with the naturally occurring ligand, S1P, regulating lymphocyte trafficking between lymphoid organs and the sites of inflammatory response. The com- 
pound also induces CD31 and $\beta$-catenin expression in subcapsular sinus endothelial cells in lymph nodes. The modulation of the inflammatory response by FTY720 may be a useful intervention in a number of inflammatory conditions by targeting bioactive sphingolipid signaling function.

\section{Cracking the Enigma of the Sphingolipids}

Distinct analysis of the biological effects mediated by variations in the key sphingolipid enzyme activity or its localization, as well as the complex and structurally diverse composition of the mediators, was hampered for a long time by the lack of an accurate and reliable methodology to measure the sphingolipid factors relevant in, for example, pro-inflammatory signaling. A plethora of papers stress the importance of distinguishing between de novo synthesized sphingolipids and degradation products derived from turnover or metabolism from more complex sphingolipids. Over the past decades, sphingolipids, e.g. ceramide, have been discovered to have not only structural but also signaling properties, especially during the stress response. The dynamic balance between sphingolipid metabolites existing in a phosphorylated or dephosphorylated as well as in an acylated or deacylated form, has been recognized as a fundamental factor determining cell fate because of its ambiguous and often opposing properties. On one hand, consistent with their functions as bioactive lipids, ceramide and its metabolites are present in very low levels in the cell's common lipid machinery. On the other hand, the interconvertibility of these mediators and the highly variable kinetics in metabolism present a difficult technical challenge for examining the absolute concentrations at operator-defined time points. This is of particular relevance when various agents for stimulation or analysis of cells consisting of a multitude of cellular subpopulations such as circulating leukocytes are tested. Biological examinations frequently focus on pro-apoptotic or cyto-protective effects alone when mediators are studied. Thus, for a more complete picture it is imperative to analyze all or at least a majority of the lipid mediators potentially responsible for the effects of interest. For this purpose, a number of relatively specific enzymatic methods have been developed based on the use of lipid kinases. These assays are relatively insensitive, time consuming, and require huge amounts of lipid material [70-72]. Derivatization and subsequent separation by high performance liquid chromatography and fluorescence detection have improved detection sensitivity $[73,74]$. The problem of co-elution of related, very similar or interfering compounds, however, has to be addressed. In addition, there is an urgent need for the preparation of internal standards for each unique mediator of interest. Methods based on metabolic labeling of the cellular sphingolipid pool with radioactive or fluorescently labeled compounds are also burdened with concerns regarding improper distribution within the cell and the cellular substructures, uneven behavior in the cellular membrane, and questionable metabolic and enzymatic properties in comparison to the naturally occurring structure of interest. Overall this may be an inefficient and inaccurate method for absolute mass level determinations [75-77].

The limitations of these 'one single lipid experiments' can now be addressed by the use of recently developed methodologies, such as liquid chromatography, coupled to subsequent tandem mass spectroscopy resulting in an approach called 'sphingolipidomics' $[78,79]$. The use of mass spectroscopy is an intriguing feature for more detailed study of numerous agonists and antagonists and to better address 
the effects of previously undetectable variations in concentrations of mediators with a sphingolipid backbone. In fact, the methods of sphingolipidomics also facilitate the determination of isotype specific effects, such as the induction of mitochondrial apoptosis exclusively by $\mathrm{C} 16: 0$ ceramide [80]. Additional information is also obtained by the analysis of the biological significance of the presence or absence of a double bond in the backbone. This difference cannot be distinguished by conventional methods due to strong structural similarity and the poor discrimination when using enzymatic methods. Saturated analogs, however, are usually biologically inactive [81]. Sphingolipidomics will provide thrilling information on the source and the 'job history' of a unique metabolite by comparative analysis of the levels of its de novo precursors versus its degradation products. The convenience of the method can also be applied for the discrimination of cellular effects of extra- or intracellular sphingolytic activity as well as for the determination of the biological effects, e.g., of a selected sphingomyelinase isoform targeted to distinct sub-cellular compartments. Such studies are mandatory for probing the impact of different intracellular sphingomyelin pools $[7,82]$. We are convinced that sphingolipidomics will provide useful information on the role, the origin, and the fate of a variety of sphingolipids orchestrating cellular signaling.

\section{Conclusion}

There is increasing evidence suggesting a pivotal role of sphingolipids as mediators regulating apoptosis, the cellular stress response, and inflammation. Key regulating enzymes in these processes are sphingomyelinases and ceramidases, generating a fine tuned 'rheostat' between lipid mediators often responsible for opposite cellular effects. The generation of knock-out models and administration of specific low molecular weight inhibitors has enabled the detailed study of the effects at a cellular level. Accumulating evidence emphasizes a critical role of ceramide in systemic inflammation mediated by a plasma secreted isoform of acid sphingomyelinase, SMPD1. An intriguing functional concept for the role of plasma secreted SMPD1 in receptor signaling activation pathways suggests that the enzyme modifies membrane fluidity by the formation of ceramide enriched rafts. Subsequent structural alterations of membrane morphology may then allow rapid and efficient signaling inside the cell, explaining the function of the enzyme in a variety of effects in cellular stress response, but also in the development of MOF during systemic inflammation. Accordingly, plasma secreted SMPD1 is hypothesized not only to function as a signaling molecule per se, but also to be involved in the host response and development of remote organ failure. However, for future therapeutic interventions, it is very important to specifically target the enzyme and the ceramide pool in the precise tissue or cell. A therapeutic intervention of ceramide generation might be envisioned to prevent tissue damage during development of organ failure and to prevent infection of mammalian cells with $P$. aeruginosa and other pathogens.

Acknowledgement: The authors thank A. C. Bunck and M. Dorer for valuable contributions during the preparation of the manuscript and our associates, students and colleagues, especially K. Reinhart and M. Bauer, for contributions to the work. Financial support by IZKF Jena to RAC is gratefully acknowledged. 


\section{References}

1. Chalfant CE, Spiegel S (2005) Sphingosine 1-phosphate and ceramide 1-phosphate: expanding roles in cell signaling. J Cell Sci 118:4605-4612

2. Baumruker T, Prieschl EE (2002) Sphingolipids and the regulation of the immune response. Semin Immunol 14:57-63

3. Pettus BJ, Chalfant CE, Hannun YA (2004) Sphingolipids in inflammation: roles and implications. Curr Mol Med 4:405-418

4. Hinkovska-Galcheva V, Boxer LA, Kindzelskii A, et al (2005) Ceramide 1-phosphate, a mediator of phagocytosis. J Biol Chem 280:26612-26621

5. Gomez-Munoz A, Kong JY, Salh B, Steinbrecher UP (2004) Ceramide-1-phosphate blocks apoptosis through inhibition of acid sphingomyelinase in macrophages. J Lipid Res 45:99-105

6. van Meer G, Lisman Q (2002) Sphingolipid transport: rafts and translocators. J Biol Chem $277: 25855-25858$

7. Birbes H, Luberto C, Hsu Y', El Bawab S, Hannun YA, Obeid LM (2005) A mitochondrial pool of sphingomyelin is involved in TNFalpha-induced Bax translocation to mitochondria. Biochem J 386:445-451

8. Paris F, Grassme H, Cremesti A, et al (2001) Natural ceramide reverses Fas resistance of acid sphingomyelinase $(-/-)$ hepatocytes. J Biol Chem 276:8297-8305

9. Heinrich M, Wickel M, Winoto-Morbach S, et al (2000) Ceramide as an activator lipid of cathepsin D. Adv Exp Med Biol 477:305-315

10. Alessenko A, Chatterjee S (1995) Neutral sphingomyelinase: localization in rat liver nuclei and involvement in regeneration/proliferation. Mol Cell Biochem 143:169-174

11. Gulbins E, Li PL (2006) Physiological and pathophysiological aspects of ceramide. Am J Physiol Regul Integr Comp Physiol 290:R11 - 26

12. Goni FM, Alonso A (2002) Sphingomyelinases: enzymology and membrane activity. FEBS Lett 531:38-46

13. Marchesini N, Hannun YA (2004) Acid and neutral sphingomyelinases: roles and mechanisms of regulation. Biochem Cell Biol 82:27-44

14. Schneider PB, Kennedy EP (1967) Sphingomyelinase in normal human spleens and in spleens from subjects with Niemann-Pick disease. J Lipid Res 8:202-209

15. Tomiuk S, Zumbansen M, Stoffel W (2000) Characterization and subcellular localization of murine and human magnesium-dependent neutral sphingomyelinase. J Biol Chem 275:5710-5717

16. Czarny M, Liu J, Oh P, Schnitzer JE (2003) Transient mechanoactivation of neutral sphingomyelinase in caveolae to generate ceramide. J Biol Chem 278:4424-4430

17. Hofmann K, Tomiuk S, Wolff G, Stoffel W (2000) Cloning and characterization of the mammalian brain-specific, Mg2+-dependent neutral sphingomyelinase. Proc Natl Acad Sci USA 97:5895-5900

18. Adam-Klages S, Schwandner R, Adam D, Kreder D, Bernardo K, Kronke M (1998) Distinct adapter proteins mediate acid versus neutral sphingomyelinase activation through the $\mathrm{p} 55$ receptor for tumor necrosis factor. J Leukoc Biol 63:678-682

19. Veldman RJ, Maestre N, Aduib OM, Medin JA, Salvayre R, Levade T (2001) A neutral sphingomyelinase resides in sphingolipid-enriched microdomains and is inhibited by the caveolinscaffolding domain: potential implications in tumour necrosis factor signalling. Biochem I 355:859-868

20. Levy M, Castillo SS, Goldkorn T (2006) nSMase2 activation and trafficking are modulated by oxidative stress to induce apoptosis. Biochem Biophys Res Commun 344:900-905

21. Tabas I (1999) Secretory sphingomyelinase. Chem Phys Lipids 102:123-130

22. Schissel SL, Keesler GA, Schuchman EH, Williams KJ, Tabas I (1998) The cellular trafficking and zinc dependence of secretory and lysosomal sphingomyelinase, two products of the acid sphingomyelinase gene. J Biol Chem 273:18250-18259

23. Dhami R, Schuchman EH (2004) Mannose 6-phosphate receptor-mediated uptake is defective in acid sphingomyelinase-deficient macrophages: implications for Niemann-Pick disease enzyme replacement therapy. J Biol Chem 279:1526-1532

24. Ni X, Morales CR (2006) The lysosomal trafficking of acid sphingomyelinase is mediated by sortilin and mannose 6-phosphate receptor. Traffic 7:889-902 
25. Marathe S, Schissel SL, Yellin MJ, et al (1998) Human vascular endothelial cells are a rich and regulatable source of secretory sphingomyelinase. Implications for early atherogenesis and ceramide-mediated cell signaling. J Biol Chem 273:4081-4088

26. Callahan JW, Jones CS, Davidson DJ, Shankaran P (1983) The active site of lysosomal sphingomyelinase: evidence for the involvement of hydrophobic and ionic groups. J Neurosci Res $10: 151-163$

27. Schissel SL, Jiang X, Tweedie-Hardman J, et al (1998) Secretory sphingomyelinase, a product of the acid sphingomyelinase gene, can hydrolyze atherogenic lipoproteins at neutral $\mathrm{pH}$. Implications for atherosclerotic lesion development. J Biol Chem 273:2738-2746

28. Niwa M, Kozawa O, Matsuno H, Kanamori Y, Hara A, Uematsu T (2000) Tumor necrosis factor-alpha-mediated signal transduction in human neutrophils: involvement of sphingomyelin metabolites in the priming effect of TNF-alpha on the fMLP-stimulated superoxide production. Life Sci 66:245-256

29. MacKinnon AC, Buckley A, Chilvers ER, Rossi AG, Haslett C, Sethi T (2002) Sphingosine kinase: a point of convergence in the action of diverse neutrophil priming agents. J Immunol 169:6394-6400

30. Prieschl EE, Csonga R, Novotny V, Kikuchi GE, Baumruker T (1999) The balance between sphingosine and sphingosine-1-phosphate is decisive for mast cell activation after Fc epsilon receptor I triggering. J Exp Med 190:1-8

31. Alemany R, Meyer zu Heringdorf D, van Koppen CJ, Jakobs KH (1999) Formyl peptide receptor signaling in HL-60 cells through sphingosine kinase. J Biol Chem 274: 3994-3999

32. Bollinger CR, Teichgraber V, Gulbins E (2005) Ceramide-enriched membrane domains. Biochim Biophys Acta 1746:284-294

33. Grassme H, Cremesti A, Kolesnick R, Gulbins E (2003) Ceramide-mediated clustering is required for CD95-DISC formation. Oncogene 22:5457-5470

34. Gulbins E (2003) Regulation of death receptor signaling and apoptosis by ceramide. Pharmacol Res 47:393-399

35. Gulbins E, Grassme H (2002) Ceramide and cell death receptor clustering. Biochim Biophys Acta 1585:139-145

36. Miyaji M, Jin ZX, Yamaoka S, et al (2005) Role of membrane sphingomyelin and ceramide in platform formation for Fas-mediated apoptosis. J Exp Med 202: 249-59.

37. Gulbins E, Dreschers S, Wilker B, Grassme H (2004) Ceramide, membrane rafts and infections. J Mol Med 82:357-363

38. Gulbins E, Kolesnick R (2003) Raft ceramide in molecular medicine. Oncogene 22:7070-7077

38. Simons K, Ehehalt R (2002) Cholesterol, lipid rafts, and disease. J Clin Invest 110:597-603

40. Grassme H, Jendrossek V, Riehle A, et al (2003) Host defense against Pseudomonas aeruginosa requires ceramide-rich membrane rafts. Nat Med 9:322-330

41. Ghosh $S$, Bhattacharyya $S$, Das $S$, et al (2001) Generation of ceramide in murine macrophages infected with Leishmania donovani alters macrophage signaling events and aids intracellular parasitic survival. Mol Cell Biochem 223:47-60

42. Grassme H, Riehle A, Wilker B, Gulbins E (2005) Rhinoviruses infect human epithelial cells via ceramide-enriched membrane platforms. J Biol Chem 280:26256-26262

43. Josephs $M$, Katan M, Rodrigues-Lima $F$ (2002) Irreversible inactivation of magnesiumdependent neutral sphingomyelinase 1 (NSM1) by peroxynitrite, a nitric oxide-derived oxidant. FEBS Lett 531:329-334

44. Won JS, Singh I (2006) Sphingolipid signaling and redox regulation. Free Radic Biol Med $40: 1875-1888$

45. Claus RA, Bunck AC, Bockmeyer CL, et al (2005) Role of increased sphingomyelinase activity in apoptosis and organ failure of patients with severe sepsis. Faseb J 19:1719-1721

46. Qiu H, Edmunds T, Baker-Malcolm J, et al (2003) Activation of human acid sphingomyelinase through modification or deletion of C-terminal cysteine. J Biol Chem 278: 32744-32752

47. Zhang DX, Yi FX, Zou AP, Li PL (2002) Role of ceramide in TNF-alpha-induced impairment of endothelium-dependent vasorelaxation in coronary arteries. Am J Physiol Heart Circ Physiol 283:H1785 - 1794

48. Scheel-Toellner D, Wang K, Craddock R, et al (2004) Reactive oxygen species limit neutrophil life span by activating death receptor signaling. Blood 104:2557-2564

49. Lightle $S$, Tosheva $R$, Lee A, et al (2003) Elevation of ceramide in serum lipoproteins during 
acute phase response in humans and mice: role of serine-palmitoyl transferase. Arch Biochem Biophys 419:120-128

50. Langmann T, Buechler C, Ries $S$, et al (1999) Transcription factors Sp1 and AP-2 mediate induction of acid sphingomyelinase during monocytic differentiation. I Lipid Res 40:870-880

51. Mathias S, Pena LA, Kolesnick RN (1998) Signal transduction of stress via ceramide. Biochem J 335:465-480

52. Drobnik W, Liebisch G, Audebert FX, et al (2003) Plasma ceramide and lysophosphatidylcholine inversely correlate with mortality in sepsis patients. J Lipid Res 44:754-761

53. Delogu G, Famularo G, Amati F, et al (1999) Ceramide concentrations in septic patients: a possible marker of multiple organ dysfunction syndrome. Crit Care Med 27:2413-2417

54. Takahashi T, Abe T, Sato T, et al (2002) Elevated sphingomyelinase and hypercytokinemia in hemophagocytic lymphohistiocytosis. J Pediatr Hematol Oncol 24:401-404

55. Kornhuber J, Medlin A, Bleich S, et al (2005) High activity of acid sphingomyelinase in major depression. J Neural Transm 112:1583-1590

56. Sathishkumar S, Boyanovsky B, Karakashian AA, et al (2005) Elevated sphingomyelinase activity and ceramide concentration in serum of patients undergoing high dose spatially fractionated radiation treatment: implications for endothelial apoptosis. Cancer Biol Ther $4: 979-986$

57. Goggel R, Winoto-Morbach S, Vielhaber G, et al (2004) PAF-mediated pulmonary edema: a new role for acid sphingomyelinase and ceramide. Nat Med 10:155-160

58. Zimmerman GA, McIntyre TM, Prescott SM, Stafforini DM (2002) The platelet-activating factor signaling system and its regulators in syndromes of inflammation and thrombosis. Crit Care Med 30: \$294-301

59. Garcia-Ruiz C, Colell A, Mari M, et al (2003) Defective TNF-alpha-mediated hepatocellular apoptosis and liver damage in acidic sphingomyelinase knockout mice. J Clin Invest 111:197-208

60. Llacuna L, Mari M, Garcia-Ruiz C, Fernandez-Checa IC, Morales A (2006) Critical role of acidic sphingomyelinase in murine hepatic ischemia-reperfusion injury. Hepatology 44 : $561-572$

61. Romiti E, Vasta V, Meacci E, et al (2000) Characterization of sphingomyelinase activity released by thrombin-stimulated platelets. Mol Cell Biochem 205:75-81

62. Bhatia R, Matsushita K, Yamakuchi M, Morrell CN, Cao W, Lowenstein CI (2004) Ceramide triggers Weibel-Palade body exocytosis. Circ Res 95:319-324

63. Loidl A, Sevcsik E, Riesenhuber G, Deigner HP, Hermetter A (2003) Oxidized phospholipids in minimally modified low density lipoprotein induce apoptotic signaling via activation of acid sphingomyelinase in arterial smooth muscle cells. J Biol Chem 278:32921-32928

64. Loidl A, Claus R, Ingolic E, Deigner HP, Hermetter A (2004) Role of ceramide in activation of stress-associated MAP kinases by minimally modified LDL in vascular smooth muscle cells. Biochim Biophys Acta 1690:150-158

65. Claus RA, Wustholz A, Muller $S$, et al (2005) Synthesis and antiapoptotic activity of a novel analogue of the neutral sphingomyelinase inhibitor scyphostatin. Chembiochem 6:726-737

66. Arenz C, Thutewohl M, Block O, Waldmann H, Altenbach HI, Giannis A (2001) Manumycin $A$ and its analogues are irreversible inhibitors of neutral sphingomyelinase. Chembiochem 2:141- 143

67. Yokomatsu T, Takechi H, Akiyama T, et al (2001) Synthesis and evaluation of a difluoromethylene analogue of sphingomyelin as an inhibitor of sphingomyelinase. Bioorg Med Chem Lett 11: $1277-12780$

68. Kolzer M, Werth N, Sandhoff K (2004) Interactions of acid sphingomyelinase and lipid bilayers in the presence of the tricyclic antidepressant desipramine. FEBS Lett 559:96-98

69. Brinkmann V, Lynch KR (2002) FTY720: targeting G-protein-coupled receptors for sphingosine 1-phosphate in transplantation and autoimmunity. Curr Opin Immunol 14:569-575

70. Hassler DF, Laethem RM, Smith GK (2000) A high throughput sphingomyelinase assay. Methods Enzymol 311:176-184

71. Olivera A, Spiegel S (1998) Sphingosine kinase. Assay and product analysis. Methods Mol Biol 105:233-242

72. Bartelsen O, Lansmann S, Nettersheim M, Lemm T, Ferlinz K, Sandhoff K (1998) Expression of recombinant human acid sphingomyelinase in insect Sf21 cells: purification, processing and enzymatic characterization. J Biotechnol 63:29-40 
73. He X, Chen F, Dagan A, Gatt S, Schuchman EH (2003) A fluorescence-based, high-performance liquid chromatographic assay to determine acid sphingomyelinase activity and diagnose types A and B Niemann-Pick disease. Anal Biochem 314:116-120

74. He X, Dagan A, Gatt S, Schuchman EH (2005) Simultaneous quantitative analysis of ceramide and sphingosine in mouse blood by naphthalene-2,3-dicarboxyaldehyde derivatization after hydrolysis with ceramidase. Anal Biochem 340:113-122

75. Liu B, Hannun YA (2000) Sphingomyelinase assay using radiolabeled substrate. Methods Enzymol 311:164-167

76. Tomas M, Duran JM, Lazaro-Dieguez F, Babia T, Renau-Piqueras I, Egea G (2004) Fluorescent analogues of plasma membrane sphingolipids are sorted to different intracellular compartments in astrocytes; Harmful effects of chronic ethanol exposure on sphingolipid trafficking and metabolism. FEBS Lett 563:59-65

77. Pagano RE, Chen CS (1998) Use of BODIPY-labeled sphingolipids to study membrane traffic along the endocytic pathway. Ann N Y Acad Sci 845:152-160

78. Bielawski J, Szulc ZM, Hannun YA, Bielawska A (2006) Simultaneous quantitative analysis of bioactive sphingolipids by high-performance liquid chromatography-tandem mass spectrometry. Methods 39:82-91

79. Merrill AH Jr, Sullards MC, Allegood JC, Kelly S, Wang E (2005) Sphingolipidomics: highthroughput, structure-specific, and quantitative analysis of sphingolipids by liquid chromatography tandem mass spectrometry. Methods 36:207-224

80. Ogretmen B, Pettus BJ, Rossi MJ, et al (2002) Biochemical mechanisms of the generation of endogenous long chain ceramide in response to exogenous short chain ceramide in the A549 human lung adenocarcinoma cell line. Role for endogenous ceramide in mediating the action of exogenous ceramide. J Biol Chem 277:12960-12969

81. Osawa Y, Uchinami H, Bielawski J, Schwabe RF, Hannun YA, Brenner DA (2005) Roles for C16-ceramide and sphingosine 1-phosphate in regulating hepatocyte apoptosis in response to tumor necrosis factor-alpha. J Biol Chem 280:27879-27887

82. Birbes H, El Bawab S, Hannun YA, Obeid LM (2001) Selective hydrolysis of a mitochondrial pool of sphingomyelin induces apoptosis. Faseb J 15: 2669-2679

83. Marchesini N, Luberto C, Hannun YA (2003) Biochemical properties of mammalian neutral sphingomyelinase 2 and its role in sphingolipid metabolism. J Biol Chem 278:13775-13783 